\title{
Treatment of patients with type 2 diabetes and non-alcoholic fatty liver disease: current approaches and future directions
}

\author{
Kenneth Cusi ${ }^{1,2}$
}

Received: 9 February 2016 / Accepted: 19 February 2016 / Published online: 21 April 2016

(C) The Author(s) 2016. This article is published with open access at Springerlink.com

\begin{abstract}
Non-alcoholic fatty liver disease (NAFLD) is reaching epidemic proportions in patients with type 2 diabetes. Patients with NAFLD are at increased risk of more aggressive liver disease (non-alcoholic steatohepatitis [NASH]) and at a higher risk of death from cirrhosis, hepatocellular carcinoma and cardiovascular disease. Dysfunctional adipose tissue and insulin resistance play an important role in the pathogenesis of NASH, creating the conditions for hepatocyte lipotoxicity. Mitochondrial defects are at the core of the paradigm linking chronic excess substrate supply, insulin resistance and NASH. Recent work indicates that patients with NASH have more severe insulin resistance and lipotoxicity compared with matched obese controls with only isolated steatosis. This review focuses on available agents and future drugs under development for the treatment of NAFLD/NASH in type 2 diabetes. Reversal of lipotoxicity with pioglitazone is associated with significant histological improvement, which occurs within 6 months and persists with continued treatment (or for at least 3 years) in patients with prediabetes or type 2 diabetes, holding potential to modify the natural history of the disease. These results also suggest that pioglitazone may become the standard of care for this population. Benefit has also been reported in non-diabetic patients. Recent promising results with glucagon-like peptide 1 receptor agonists have opened another new treatment avenue for NASH. Many
\end{abstract}

\section{Kenneth Cusi \\ Kenneth.Cusi@medicine.ufl.edu}

1 Division of Endocrinology, Diabetes and Metabolism, University of Florida, 1600 SW Archer Road, Room H-2, Gainesville, FL 32610, USA

2 Malcom Randall Veterans Administration Medical Center, Gainesville, FL, USA agents in Phase 2-3 of development are being tested, aiming to restore glucose/lipid metabolism, ameliorate adipose tissue and liver inflammation, or to inhibit liver fibrosis. By targeting a diversity of relevant pathways, combination therapy in NASH will likely provide greater success in the future. In summary, increased clinical awareness and improved screening strategies (as currently done for diabetic retinopathy and nephropathy) are needed, to translate recent treatment progress into early treatment and improved quality of life for patients with type 2 diabetes and NASH. This review summarises a presentation given at the symposium 'The liver in focus' at the 2015 annual meeting of the EASD. It is accompanied by two other reviews on topics from this symposium (by John Jones, DOI: 10.1007/s00125-016-3940-5, and by Hannele Yki-Järvinen, DOI: 10.1007/s00125-016-3944-1) and a commentary by the Session Chair, Michael Roden (DOI: 10.1007/s00125-016-3911-x).

Keywords Fatty liver - Insulin resistance $\cdot$ NAFLD $\cdot$ NASH · Non-alcoholic steatohepatitis · Pioglitazone · Prediabetes . Treatment · Type 2 diabetes

$\begin{array}{ll}\text { Abbreviations } \\ \text { AST } & \text { Aspartate aminotransferase } \\ \text { CCR } & \text { C-C chemokine receptor } \\ \text { CVD } & \text { Cardiovascular disease } \\ \text { GLP-1 } & \text { Glucagon-like peptide 1 } \\ \text { GLP-1RAs } & \text { GLP-1 receptor agonists } \\ \text { HCC } & \text { Hepatocellular carcinoma } \\ \text { NAFLD } & \text { Non-alcoholic fatty liver disease } \\ \text { NAS } & \text { NAFLD activity score } \\ \text { NASH } & \text { Non-alcoholic steatohepatitis } \\ \text { PPAR } & \text { Peroxisome proliferator-activated receptor } \\ \text { RCT } & \text { Randomised controlled trial }\end{array}$


SGLT2 Sodium-glucose cotransporter 2

TCA Tricarboxylic acid

TZD Thiazolidinediones

\section{Why treat NASH in patients with type 2 diabetes?}

Non-alcoholic fatty liver disease (NAFLD) is a frequent comorbidity in both paediatric and adult populations, in particular in the setting of obesity and type 2 diabetes [1-3]. It is estimated that between 75 million to 100 million individuals in the USA may have NAFLD [2], with high rates also reported worldwide [1]. The magnitude of the epidemic will make screening imperative, particularly in obese patients with type 2 diabetes, who are at the highest risk of developing its more aggressive form with hepatocyte injury (NASH). Patients with diabetes are also at a higher risk of fibrosis, end-stage liver disease and hepatocellular carcinoma (HCC), as well as extra-hepatic complications [4]. However, few studies have systematically screened patients with type 2 diabetes. In our experience, about $70 \%$ of obese patients with diabetes have NAFLD and as many as $30-40 \%$ have NASH [5-7]. The prevalence of both remains high even when plasma aminotransferase concentration is normal, with about half having steatosis (when measured by proton magnetic resonance imaging and spectroscopy $\left[{ }^{1} \mathrm{H}-\mathrm{MRS}\right]$ ), about one-third having NASH and many early fibrosis [8]. Other investigators have reported similarly high rates of steatosis $(\sim 70 \%)$ [9-12] and fibrosis (17-55\%) [11-13]. The results of two recent large screening studies (one from Hong Kong [ $n=1,918]$ [13] and another from Rotterdam [ $n=3,041]$ [14]) were consistent with this, reporting that fibrosis affects one out of every six middle-aged patients with diabetes. Of note, on histology, isolated steatosis (i.e. without features of hepatocyte necrosis or inflammation) is no longer considered a 'benign' condition, at least in type 2 diabetes, as emerging evidence indicates that many patients with isolated steatosis develop hepatocyte injury and fibrosis over time [15]. Liver fibrosis is the single best predictor of future cirrhosis $[16,17]$ and it occurs much more frequently in diabetes [18].

It should also be noted that NAFLD is becoming a major cause of HCC in the USA. A recent study reported that between 2004 and 2009, HCC related to NASH increased by $9 \%$ annually and was associated with shorter survival time compared with other predisposing aetiologies [19]. Lack of systematic screening and treatment for NASH, even among hepatologists [20], has led to it being massively underdiagnosed, which explains why NASH is the second largest cause of cirrhosis and liver transplantation in the USA [21].

Another major reason for addressing NAFLD in diabetes is its strong association with cardiovascular disease (CVD) $[1,2$, 22]. While most physicians place a high priority on preventing macrovascular complications in type 2 diabetes, few are aware that the presence of NAFLD appears to significantly increase the risk. Although the nature of this association remains a subject of intense investigation, there is good evidence that NAFLD promotes dyslipidaemia [23], hyperinsulinaemia [24] and subclinical inflammation [1, 2, 22], all of which are potentially atherogenic risk factors. The presence of NAFLD may also worsen microvascular disease and other comorbidities often present in diabetes $[1,4]$.

In summary, patients with type 2 diabetes who also have NASH appear to be at a significantly higher risk of death from either cirrhosis, HCC and/or CVD. A specific screening strategy for this population must be developed and implemented, as has been done for diabetic microvascular complications [1, $25]$, and included in future guidelines [26, 27].

\section{Available pharmacological agents for the treatment of NASH in patients with type 2 diabetes}

Insulin-sensitisers in NASH Restoring insulin action is a major treatment target given the central role of adipose tissue insulin resistance and 'lipotoxicity' in the pathogenesis of NASH [1]. Insulin sensitivity and hepatic steatosis usually improve with lifestyle intervention in proportion to the magnitude of weight loss. Significant reversal of hepatocyte injury requires weight loss of about $10 \%[1,2,28]$. Bariatric surgery also improves NASH [28]. In contrast, long-term improvement of fibrosis by either lifestyle intervention or bariatric surgery is uncertain owing to a lack of controlled prospective studies beyond 12 months' duration (reviewed in [26, 28]).

Insulin-sensitisers have been widely tested in NAFLD. Early open-label studies with metformin suggested a histological benefit $[29,30]$. However, a specific effect of the biguanide was difficult to establish given the larger than expected associated weight loss in these studies, while more recent randomised controlled trials (RCTs) have yielded negative results in both children [31] and adults (reviewed in [1,3, $26,28])$. While the aetiology of NASH is multifactorial, unabated lipolysis in dysfunctional/insulin-resistant adipose plays a key role by promoting ectopic triacylglycerol accumulation in many tissues [1-3]. Adipose tissue dysfunction and hepatic insulin resistance are more severe in diabetic patients with NASH compared with matched obese controls with only isolated steatosis [32], and mitochondrial defects appear to be at the core of the paradigm linking excess substrate supply, insulin resistance, lipotoxicity and NASH [33]. Recent studies from our laboratory [34, 35], and others [36-38], support this view. In animal models of diet-induced NASH (C57BL/6 mice fed a high-trans-fat, high-fructose diet for 24 weeks) hepatic mitochondrial fluxes are increased, but not enough to prevent the formation of inflammatory cytokines, lipid peroxidation products and other toxic lipid metabolites (ceramides, diacylglycerols and acylcarnitines, among others), suggesting inadequate adaptation with inefficient 
disposal of excess fatty acids through the tricarboxylic acid (TCA) cycle [34]. Recent human studies indicate inadequate mitochondrial adaptation despite increased TCA cycle activity in obese patients with hepatic steatosis [39]. In patients with $\mathrm{NASH}$, mitochondrial mass is greater but is characterised by proton leakage, reduced antioxidant capacity and more prone to trigger inflammation [38]. In these provocative studies only a handful of patients were studied, so much more remains to be learned in relation to factors such as ethnicity, presence or absence of diabetes, ageing, genetic polymorphisms (e.g. PNLPA3, TM6SF2) and response to weight loss or pharmacotherapy. It has been established that mitochondrial oxidative capacity must retain its ability to be highly adaptable (retain 'metabolic flexibility') to changing metabolic demands in order to prevent cell injury and activation of apoptotic pathways. Under conditions of chronic overfeeding, as observed in obesity or type 2 diabetes, metabolic flexibility is lost. This hypothesis clearly requires further experimental validation. However, recent proof-of-concept experimental approaches that relieve mitochondrial demand either by weight loss or hormonal manipulation [40], or more specifically, by mild mitochondrial uncoupling using the protonophore 2,4-dinitrophenol (DNP) [36], reverse hepatic triacylglycerol accumulation and NASH.

Within this context, thiazolidinediones (TZDs) are ligands that target the transcription factor peroxisome proliferatoractivated receptor (PPAR)- $\gamma$, with broad effects on glucose/ lipid metabolism through modulation of substrate supply, insulin signalling and mitochondrial fatty acid oxidation [1, 41]. In humans with NASH, pioglitazone enhances insulin sensitivity and prevents excessive rates of lipolysis [42-44]. The positive effects of PPAR- $\gamma$ action on adipocyte biology translate into a two- to threefold increase in plasma adiponectin concentration [44]. In recent work, Bril et al [45] reported that the response to pioglitazone in NASH can be predicted by the increase in plasma adiponectin levels within the first 1-3 months after treatment initiation. It has been about a decade since Belfort et al [42] first reported in a proof-of-concept study that in patients with NASH and either impaired glucose tolerance or type 2 diabetes, pioglitazone treatment significantly ameliorated hepatic steatosis and necroinflammation. The NAS improved for most participants, while treatment difference in resolution of NASH vs placebo occured in about one-third. Pioglitazone also reduced liver fibrosis compared with baseline $(p=0.002)$, but not when compared with placebo $(p=0.08)$. This RCT was important in establishing that NASH could be reversed within a relatively short period of time (6 months). Pioglitazone also prevents the progression from impaired glucose tolerance to diabetes [46], and one may speculate that this effect is tied to its ability to reverse NAFLD/NASH [47]. Histological improvement with pioglitazone in NASH was later also reported in patients without diabetes [48, 49]. Recently, a 3 year study in 101 patients with NASH and either impaired fasting glucose and/or impaired glucose tolerance or type 2 diabetes confirmed its long-term safety and efficacy in this population (K. Cusi, B. Orsak, F. Bril, R. Lomonaco, J. Hecht, C. Ortiz-Lopez, unpublished results). Taken together, these results suggest that pioglitazone may modify the natural history of the disease. No patient discontinued the trial due to a pioglitazone-induced adverse event and weight gain was modest overall ( $3 \mathrm{~kg}$ over 3 years). Weight gain with pioglitazone treatment is primarily due to improved insulin action and enhanced adipocyte triacylglycerol storage, and less often the result of fluid retention [50, 51]. Pioglitazone decreases cardiovascular events [1,41], a feature frequently forgotten but of value in this population. However, development of shortness of breath or congestive heart failure, while rare, may occur in patients with type 2 diabetes and NAFLD with undiagnosed diastolic dysfunction [52]. Bone loss may occur with TZD treatment in women, and pioglitazone should not be used in pregnancy or in children or adolescents [53]. A 10 year prospective study showed no association between pioglitazone and bladder cancer [54].

Glucagon-like peptide 1 receptor agonists and dipeptidyl peptidase 4 inhibitors Indirect evidence from animal studies (reviewed in $[1,55]$ ), and recent meta-analysis of clinical trials of liraglutide in type 2 diabetes [56], have suggested that glucagon-like peptide 1 receptor agonists (GLP-1RAs) could improve NASH. However, clear evidence was lacking until the landmark proof-of-concept study by Armstrong et al [57]. The investigators treated 52 obese insulin-resistant patients, one-third of whom had type 2 diabetes, with liraglutide or placebo for 48 weeks. Resolution of NASH was observed in $39 \%$ of liraglutide-treated patients compared with $9 \%$ of patients in the placebo group (RR 4.3, 95\% CI 1.0, 17.7; $p=0^{\circ} 019$ ), a treatment difference of $\sim 30 \%$. In addition, there was less progression of liver fibrosis over time in the treatment group $(p<0.05)$. Liver and adipose tissue insulin sensitivity improved in a subset of patients that underwent careful glucose turnover studies [58]. These results have created significant excitement for additional GLP-1RA studies in NASH to understand the relative contribution of unspecific mechanisms such as changes in weight, insulin sensitivity or glucotoxicity [59] vs effects on specific hepatocyte GLP-1 signalling pathways $[60,61]$.

In contrast, studies with dipeptidyl peptidase 4 (DPP-4) inhibitors have reported mixed results. Sitagliptin has been reported to be neutral [62] or to decrease [63] plasma aminotransferases, although their overall impact on liver histology is unknown. In diabetic patients with mild steatosis, Macauley et al [64] observed a small but significant reduction (from $7.3 \%$ to $5.3 \%$, normal $\leq 5.5 \% ; p=0.001$ ) in hepatic triacylglycerol accumulation with vildagliptin treatment for 6 months, a change that correlated closely with the decrease in fasting plasma concentration. Thus, hyperglycaemia may be an important factor that promotes hepatic steatosis, as 
suggested in cross-sectional studies [8, 32], although the role of reversing glucotoxicity per se on NASH (independent of treating insulin resistance) has never been examined in an RCT in type 2 diabetes.

\section{Sodium-glucose cotransporter 2 (SGLT2) inhibitors} Control of hyperglycaemia by inhibition of proximal tubule glucose reabsorption [65] is actively being investigated as a potential approach for NAFLD in patients with type 2 diabetes. A reduction in intrahepatic triacylglycerol accumulation would be expected from a decrease in substrate supply to the liver by the combined effects of normoglycaemia plus modest weight loss and enhanced insulin sensitivity. In animal models of NASH, sodium-glucose cotransporter 2 (SGLT2) inhibitors have unique antifibrotic properties [66]. In patients with diabetes, levels of plasma aminotransferases decrease during treatment with SGLT2 $[67,68]$. Recently, pooled data from four 26 week placebo-controlled studies of canagliflozin $(n=2313)$ and two 52 week active-controlled studies of canagliflozin vs sitagliptin $(n=1488)$ found significant reductions in plasma alanine aminotransferase (ALT) with canagliflozin $300 \mathrm{mg}$ compared with placebo or sitagliptin [69]. Changes in aspartate aminotransferase (AST)/ALT were fully explained by the reduction in $\mathrm{HbA}_{1 \mathrm{c}}$ and body weight. Future studies are needed to define the role of SGLT2 inhibitors in the management of patients with NAFLD and type 2 diabetes.

\section{Other therapeutic agents tested for the treatment of NASH}

Many agents have been tested for the treatment of NASH $[1-3,28,55]$ but few have included a significant number of patients with type 2 diabetes. Most have not improved liver histology. Lipid-lowering agents have been tested extensively and can be used safely in this population [26, 27], but statins [70], ezetimibe [71], fibrates [72], niacin [72], omega-3 polyunsaturated fatty acids (PUFAs) [73] and colesevelam [74] do not improve steatosis to a clinically meaningful extent (or hepatocyte injury when examined; reviewed in $[28,55]$ ). Instead, amelioration of hepatocyte injury has been reported with vitamin $\mathrm{E}$ in adults without type 2 diabetes at doses of $800 \mathrm{IU} /$ day [49]. However, resolution of NASH was of borderline significance ( $36 \%$ vs $21 \%, p=0.05$ vs placebo) in contrast to the effect of pioglitazone in the same trial $(47 \%$ vs $21 \%, p=0.001$ vs placebo) [49]. In a controlled trial in nondiabetic children with NASH, treatment with vitamin $\mathrm{E}$ or metformin had overall negative effects [31]. Vitamin E may decrease intracellular oxidative stress, although the mechanism of action is unclear. The long-term safety of vitamin $\mathrm{E}$ in patients with NASH remains to be established given its controversial increase in the risk of prostate cancer with long-term exposure [75]. An ongoing RCT will soon establish its role in patients with type 2 diabetes, either alone or combined with pioglitazone (NCT01002547). Finally, pentoxifylline, a TNF- $\alpha$ agonist and non-selective phosphodiesterase inhibitor, has been studied in NASH in two small controlled 12 month studies. Van Wagner et al [76] reported no histological improvement in 30 patients, while another small trial $(n=26)$ observed a modest benefit on steatosis, lobular inflammation and fibrosis [77]. Larger studies are clearly needed to establish its role in NASH.

\section{Future treatments for patients with NASH}

From the previous sections it is evident that additional agents are needed for the treatment of NASH. Moreover, no existing agent tested has been specifically approved to this end. This has led to an explosion of agents currently under investigation. Importantly, most studies now include a large number of patients with type 2 diabetes given their higher risk of disease progression.

Many approaches have been tested. Table 1 includes agents at more advanced stages of development (Phase $2 \mathrm{a} / 2 \mathrm{~b}$ or early Phase 3 trials). But the challenge ahead is substantial. For example, attempts to control triacylglycerol synthesis, such as with $11 \beta$-hydroxysteroid dehydrogenase type 1 (11ß-HSD1) [78] or stearoyl coenzyme A desaturase (SCD)-1 pathways [79] have led to overall discouraging results. Greater success has been achieved by targeting PPAR $\alpha / \operatorname{PPAR} \delta$ with the dual agonist elafibranor (GFT505) [80], a partial PPAR $\alpha$ agonist with about $60 \%$ of the maximal response $\left(E_{\max }\right)$ of fenofibrate [81]. Dual PPAR $\alpha$ and PPAR $\delta$ activation mitigates inflammation and improves hepatic insulin sensitivity in vivo [82] and in insulin-resistant patients [83]. In contrast, the PPAR $\alpha$ agonist fenofibrate has not been associated with a decrease in insulin resistance or plasma aminotransferase concentration in patients with the metabolic syndrome [84] or decreased hepatic steatosis in patients with NAFLD [72]. Despite the above strong rationale, discouraging results were reported after 52 weeks of elafibranor treatment in 276 patients with biopsy-proven NASH ( $\sim 40 \%$ with type 2 diabetes) as it failed to meet the primary outcome of resolution of NASH without worsening of fibrosis [84]. This was, at least in part, due to many patients having borderline NASH on liver histology before treatment. However, resolution of NASH ( $20 \%$ vs $11 \%, p=0.018)$ and improved scores of hepatocyte injury and fibrosis were noted at the higher dose $(120 \mathrm{mg} /$ day $)$ in patients with more severe disease at baseline (NAFLD activity score [NAS] $\geq 4 ; n=234$ ) [85]. These results have been the basis for an ongoing large multicentre RCT with elafibranor enrolling only patients with more advanced liver disease (NAS $\geq 4$ ) (NCT02704403) [85].

A different strategy involves more directly targeting inflammation by inhibition of C-C chemokine receptors type 2 (CCR2) and type 5 (CCR5), which mediate monocyte and macrophage infiltration and inflammation in adipose tissue $[86,87]$. Overexpression of CCR2 and CCR5 receptors and 
Table 1 Pharmaceutical agents under development for the treatment of NASH

\begin{tabular}{|c|c|c|c|}
\hline Therapeutic agent & Manufacturer & Target & Proposed mode of action \\
\hline BMS986036 & Bristol-Myers Squibb & Modulation of FGF21 metabolism & $\begin{array}{l}\text { Improvement of hepatic lipid and glucose } \\
\text { metabolism; anti-inflammatory }\end{array}$ \\
\hline Cenicriviroc & Tobira Therapeutics & CCR 2 and CCR5 & $\begin{array}{l}\text { Inhibition of CCR2- and CCR5-mediated } \\
\text { monocyte/macrophage infiltration and inflammation }\end{array}$ \\
\hline Elafibranor & Genfit & $\begin{array}{l}\text { Modulation of hepatic PPAR } \alpha \\
\text { and PPAR } \delta \text { pathways }\end{array}$ & $\begin{array}{l}\text { Stimulation of NEFA oxidation; improvement of } \\
\text { lipid and glucose metabolism; prevention } \\
\text { of inflammation }\end{array}$ \\
\hline Emricasan & Conatus Pharmaceuticals & Caspase pathways (pan-caspase inhibitor) & $\begin{array}{l}\text { Inhibition of fibrosis by blocking caspase } \\
\text { protease activation and apoptosis pathways }\end{array}$ \\
\hline GR-MD-02 & Galectin Therapeutics & Galectin-3 inhibitor & Prevention of inflammation and fibrosis \\
\hline Obeticholic acid & Intercept Pharmaceuticals & FXR agonist & Regulation of hepatic glucose and lipid metabolism \\
\hline Px-104 & $\begin{array}{l}\text { Phenex Pharmaceuticals/ } \\
\text { Gilead Sciences }\end{array}$ & FXR agonist & Regulation of hepatic glucose and lipid metabolism \\
\hline Simtuzumab & Gilead Sciences & LOXL2 enzyme activity & Inhibition of fibrosis by a LOXL 2 monoclonal antibody \\
\hline
\end{tabular}

Agents are listed in alphabetical order

FXR, Farnesoid X receptor; LOXL2, Lysyl oxidase-like 2

their ligands in adipose tissue of obese patients is associated with increased inflammation and insulin resistance. Cenicriviroc is a potent inhibitor of ligand binding of CCR2 and CCR5 with antifibrotic properties in animal models of liver/kidney fibrosis. Post hoc analysis of Phase $2 b$ studies in HIV-1 patients revealed that cenicriviroc treatment improved plasma biomarkers of fibrosis (AST to Platelet Ratio Index [APRI] and Fibrosis 4 calculator [FIB-4] scores). Its mechanism of action in humans is being actively studied in obese, insulin-resistant individuals at risk of NAFLD (ORION study; NCT02330549), and in a large controlled multicentre trial in patients with NASH who are at increased risk of disease progression due to the presence of $\geq 1$ risk factor such as the metabolic syndrome, type 2 diabetes or hepatic fibrosis (CENTAUR; NCT02217475) [88].

Given that fibrosis is the primary determinant of long-term adverse outcomes [16, 17], several novel agents are specifically targeting fibrosis-related pathways. Approaches include inhibition of apoptotic pathways with the pan-caspase inhibitor emricasan, a compound that has been recently granted fast track designation by the United States Food and Drug Administration and is being tested in a Phase $2 b$ clinical trial in NASH cirrhosis (EmricasaN, a Caspase inhibitOR, for Evaluation [ENCORE-NF]). Another strategy is treatment of advanced fibrosis targeting galectins, a family of proteins with high affinity to galactose-containing glycoproteins present on cell surfaces and the extracellular matrix [89]. Galectin-3 protein is highly expressed in immune cells and has been associated with inflammation and fibrosis in several disease models, including hepatic fibrosis. A Phase 2a multicentre trial is underway in individuals with portal hypertension and NASH cirrhosis (NCT02462967). Other approaches in Phase 2 studies aimed at controlling fibrogenesis include targeting growth and inflammation through the fibroblast growth factor 21 (FGF21) pathway [90] (compound BMS986036, NCT02413372), or preventing collagen cross-linking by inhibiting lysyl oxidase-like 2 (LOXL2) enzyme activity with the monoclonal antibody simtuzumab (being tested in NASH patients with [NCT01672879] or without [NCT01672866] cirrhosis).

Finally, histological improvement may be achieved in NASH by modulation of hepatic glucose and lipid metabolism through farnesoid X receptor (FXR) pathways [91], as recently shown with the bile acid derivative 6-ethylchenodeoxycholic acid (obeticholic acid) [92]. The primary outcome of a decrease in the NAS by $\geq 2$ without worsening of fibrosis was reached in $45 \%$ of patients treated with obeticholic acid vs $21 \%$ on placebo (RR 1.9, 95\% CI 1.3, 2.8; $p=0.0002$; treatment effect $\sim 24 \%$ ). However, to reach this prespecified primary endpoint, change in the NAS did not have to necessarily include ballooning or two separate NAS variables. This departure from prior trials by the NASH Clinical Research Network [31, 49], in part aimed at simplifying the primary outcome, has made comparisons with other RCTs difficult $[31,49,84]$. While resolution of NASH did not change significantly ( $22 \%$ vs $13 \%$ on placebo, $p=0.08$ ), individual histological outcomes improved compared with placebo: steatosis $61 \%$ vs $38 \%$ ( $p=0.001)$, inflammation $53 \%$ vs $35 \%(p=0.006)$, ballooning $46 \%$ vs $31 \%(p=0.03)$ and fibrosis $35 \%$ vs $19 \%$ ( $p=0.004)$, giving a treatment difference vs placebo of between $\sim 15 \%$ and $23 \%$. There has been some concern about the increase from baseline in plasma LDL-cholesterol and the reduction in HDL-cholesterol levels, as well as the observed worsening of insulin sensitivity (according to HOMA-IR). Of note, changes in fasting plasma insulin during the trial were confounded, at least in part, by patients with diabetes on exogenous insulin during the study. However, a modest improvement in hepatic insulin sensitivity is observed with short-term 
use of obeticholic acid [93] at the daily dose of $25 \mathrm{mg}$ used in the Farnesoid X Receptor Ligand Obeticholic Acid in NASH Treatment (FLINT) [92]. A larger multicentre study is underway to fully assess its long-term efficacy and safety.

\section{Conclusions: perspective for the future}

Today there is a much greater awareness of the severe metabolic and liver-specific complications associated with NASH. Within this context, there is growing consensus to replace the acronym 'NASH', a disease of 'not being alcoholic steatohepatitis' with a more descriptive name that would help clinicians conceptually grasp the condition to be diagnosed and treated [1, 94, 95]. As reviewed earlier, there are many intracellular pathways that contribute to hepatocyte injury, and the heterogeneous nature of the disease makes a unifying name difficult. However, this problem is also true for most chronic diseases, including type 2 diabetes. A name that highlights the central role of hepatocyte lipotoxicity secondary to adipose tissue insulin resistance characteristic of obesity would be fitting. Lipotoxicity primes cells to mitochondrial dysfunction and makes them more vulnerable to toxic lipid metabolites, oxidative stress and activation of multiple inflammatory pathways $[1,94]$. Future work needs to deepen our understanding of the crosstalk between liver and adipose tissue, but the name 'lipotoxic liver disease' would encompass a significant aspect of the disease and serve as a reminder to clinicians about the important role of dysregulated adipose tissue in NAFLD.

Specific screening and treatment recommendations for patients with type 2 diabetes and NASH will need to be developed and actively pursued in the clinic. Screening for NASH in type 2 diabetes, particularly in obese patients, should be encouraged as is currently done for microvascular complications of diabetes such as retinopathy, neuropathy and nephropathy. Only early intervention is likely to modify the natural history of the disease and halt the growing epidemic of NASH [21]. Recent studies support such an approach, as one in six middle-aged patients with type 2 diabetes have liver fibrosis [13, 14, 25]. There is recent evidence of a long-term safe and effective treatment for this population in pioglitazone (K. Cusi et al, unpublished results), which will become to NASH what metformin is to type 2 diabetes today - first-line therapy and the background agent for combination strategies. This should not undermine the search for other pharmacological agents targeting the many dysfunctional pathways in NASH discussed above. Of note, drug development should target resolution of NASH, not isolated steatosis, although in early exploratory studies significant reduction in liver triacylglycerol content on imaging $\left({ }^{1} \mathrm{H}-\mathrm{MRS}\right)$ may potentially predict improvement in NASH. The magnitude of improvement on ${ }^{1} \mathrm{H}-$ MRS imaging that translates into resolution of NASH is unknown at the present time, but is likely to depend on the mechanism of action of a specific compound. As new agents become available, it is likely that combination therapy will become widely accepted for such a heterogeneous disease such as NASH, as is common practice for the treatment of type 2 diabetes or hypertension.

Future studies should explore the role of glycaemic control per se in NASH, as has been investigated for microvascular complications of diabetes. No trial to date has addressed this issue. There is also a need for long-term RCTs on the optimal lifestyle intervention, how to improve adherence and on the best macronutrient composition for NASH in type 2 diabetes. The additional role of weight loss agents and of bariatric surgery need to be better defined prospectively in controlled studies. Drug development trials will need more consistency in their trial design and primary outcomes, as well as improved research networks for efficient recruitment into large multicentre RCTs. Better standardisation across imaging methodologies and biomarkers, with better correlation/validation with histology, will be also imperative for the screening of large cohorts and the assessment of treatment response.

In summary, while the diagnosis of NAFLD/NASH today remains a challenge, screening with a combination of validated novel genetic, imaging and plasma biomarkers will in the near future facilitate management. We are at an exciting time where new awareness about the impact of NASH will prompt earlier diagnosis and treatments that for the first time may alter the natural history of the disease and improve the quality of life of millions of patients.

Funding Work by the author is supported by a VA Merit Award (1 I01 CX000167-01).

Duality of interest Kenneth Cusi (KC) has received research grant support from Janssen Pharmaceutical Co. of Johnson \& Johnson and Novartis Pharmaceuticals, and is a consultant for Janssen Pharmaceutical Co. of Johnson \& Johnson, Eli Lilly and Company, and Tobira Therapeutics, Inc.

Contribution statement The author was the sole contributor to this paper.

Open Access This article is distributed under the terms of the Creative Commons Attribution 4.0 International License (http:// creativecommons.org/licenses/by/4.0/), which permits unrestricted use, distribution, and reproduction in any medium, provided you give appropriate credit to the original author(s) and the source, provide a link to the Creative Commons license, and indicate if changes were made.

\section{References}

1. Cusi K (2012) Role of obesity and lipotoxicity in the development of nonalcoholic steatohepatitis: pathophysiology and clinical implications. Gastroenterology 142:711-725

2. Rinella ME (2015) Nonalcoholic fatty liver disease: a systematic review. JAMA 313:2263-2273 
3. Ahmed A, Wong RJ, Harrison SA. Nonalcoholic fatty liver disease review: diagnosis, treatment and outcomes. Clin Gastroenterol Hepatol 13:2062-2070

4. Armstrong MJ, Adams LA, Canbay A, Syn W-K (2014) Extrahepatic complications of nonalcoholic fatty liver disease. Hepatology 59:1174-1197

5. Lomonaco R, Ortiz-Lopez C, Orsak B et al (2011) Role of ethnicity in overweight and obese patients with nonalcoholic steatohepatitis. Hepatology 54:837-845

6. Maximos M, Bril F, Portillo-Sanchez P et al (2015) The role of liver fat and insulin resistance as determinants of plasma aminotransferase elevation in nonalcoholic fatty liver disease. Hepatology 61: 153-160

7. Bril F, Ortiz-Lopez C, Lomonaco R et al (2015) Clinical value of liver ultrasound for the diagnosis of nonalcoholic fatty liver disease in overweight and obese patients. Liver Int 35:2139-2146

8. Portillo-Sanchez P, Bril F, Maximos M et al (2015) High prevalence of nonalcoholic fatty liver disease in patients with type 2 diabetes mellitus and normal plasma aminotransferase levels. J Clin Endocrinol Metab 100:2231-2238

9. Targher G, Bertolini L, Padovani R et al (2007) Prevalence of nonalcoholic fatty liver disease and its association with cardiovascular disease among type 2 diabetic patients. Diabetes Care 30:12121218

10. Leite NC, Salles GF, Araujo AL, Villela-Nogueira CA, Cardoso CR (2009) Prevalence and associated factors of non-alcoholic fatty liver disease in patients with type-2 diabetes mellitus. Liver Int 29:113119

11. Leite NC, Villela-Nogueira CA, Pannain VL et al (2011) Histopathological stages of nonalcoholic fatty liver disease in type 2 diabetes: prevalences and correlated factors. Liver Int 31:700706

12. Casey SP, Kemp WW, McLean CA, Topliss DJ, Adams LA, Roberts SK (2012) A prospective evaluation of the role of transient elastography for the detection of hepatic fibrosis in type 2 diabetes without overt liver disease. Scand J Gastroenterol 47:836-841

13. Kwok R, Choi KC, Wong GL et al (2015) Screening diabetic patients for nonalcoholic fatty liver disease with controlled attenuation parameter and liver stiffness measurements: a prospective cohort study. Gut. doi:10.1136/gutjnl-2015-309265

14. Koehler EM, Plompen EP, Schouten JN, Hansen BE, Darwish Murad S, Taimr P et al (2016) Presence of diabetes mellitus and steatosis is associated with liver stiffness in a general population: the Rotterdam study. Hepatology 63:138-147

15. Pais R, Charlotte F, Fedchuk L et al (2013) A systematic review of follow-up biopsies reveals disease progression in patients with nonalcoholic fatty liver. J Hepatol 59:550-556

16. Ekstedt M, Hagstrom H, Nasr P et al (2015) Fibrosis stage is the strongest predictor for disease-specific mortality in NAFLD after up to 33 years of follow-up. Hepatology 61:1547-1554

17. Angulo P, Kleiner DE, Dam-Larsen S et al (2015) Liver fibrosis, but no other histologic features, is associated with long-term outcomes of patients with nonalcoholic fatty liver disease. Gastroenterology 149:389.e310-397.e310

18. Raff EJ, Kakati D, Bloomer JR, Shoreibah M, Rasheed K, Singal AK (2015) Diabetes mellitus predicts occurrence of cirrhosis an hepatocellular cancer in alcoholic liver and non-alcoholic fatty liver diseases. J Clin Transl Hepatol 3:9-16

19. Younossi Z, Otgonsuren M, Henry L et al (2015) Association of nonalcoholic fatty liver disease (NAFLD) with hepatocellular carcinoma (HCC) in the United States from 2004 to 2009. Hepatology 62:1723-1730

20. Rinella ME, Lominadze Z, Loomba R et al (2016) Practice patterns in NAFLD and NASH: real life differs from published guidelines. Ther Adv Gastroenterol 9:4-12
21. Wong RJ, Aguilar M, Cheung R et al (2015) Nonalcoholic steatohepatitis is the second leading etiology of liver diseaseamong adults awaiting liver transplantation in the United States. Gastroenterology 48:547-555

22. Lonardo A, Sookoian S, Pirola CJ, Targher G (2015) Non-alcoholic fatty liver disease and risk of cardiovascular disease. Metabolism. doi:10.1016/j.metabol.2015.09.017

23. Bril F, Sninsky JJ, Baca AM et al (2016) Hepatic steatosis and insulin resistance, but not steatohepatitis, promote atherogenic dyslipidemia in NAFLD. J Clin Endocrinol Metab 101:644-652

24. Bril F, Lomonaco R, Orsak B et al (2015) Relationship between disease severity, hyperinsulinemia, and impaired insulin clearance in patients with nonalcoholic steatohepatitis. Hepatology 59:21782187

25. Ahmed A, Perumpail RB, Harrison SA (2016) High prevalence of hepatic fibrosis in the setting of coexisting diabetes and hepatic steatosis: a case for selective screening in the general population? Hepatology 63:20-22

26. Chalasani N, Younossi Z, Lavine JE et al (2012) The diagnosis and management of non-alcoholic fatty liver disease: practice guideline by the American Association for the Study of Liver Diseases, American College of Gastroenterology, and the American Gastroenterological Association. Gastroenterology 142:1592-1609

27. European Association for the Study of the Liver (EASL), European Association for the Study of Diabetes (EASD) and European Association for the Study of Obesity (EASO) (2016) EASLEASD-EASO Clinical Practice Guidelines for the management of non-alcoholic fatty liver disease. Diabetologia. doi:10.1007/ s00125-016-3902-y

28. Lomonaco R, Sunny NE, Bril F, Cusi K (2013) Nonalcoholic fatty liver disease: current issues and novel treatment approaches. Drugs 73:1-14

29. Bugianesi E, Gentilcore E, Manini R et al (2005) A randomized controlled trial of metformin versus vitamin $\mathrm{E}$ or prescriptive diet in nonalcoholic fatty liver disease. Am J Gastroenterol 100:10821090

30. Loomba R, Lutchman G, Kleiner DE et al (2009) Clinical trial: pilot study of metformin for the treatment of non-alcoholic steatohepatitis. Aliment Pharmacol Ther 29:172-182

31. Lavine JE, Schwimmer JB, Van Natta ML et al (2011) Effect of vitamin $\mathrm{E}$ or metformin for treatment of nonalcoholic fatty liver disease in children and adolescents: the TONIC randomized controlled trial. JAMA 305:1659-1668

32. Lomonaco R, Bril F, Portillo-Sanchez P et al (2016) Metabolic impact of nonalcoholic steatohepatitis (NASH) in obese patients with type 2 diabetes mellitus. Diabetes Care. doi:10. 2337/dc15-1876

33. Begriche K, Massart J, Robin MA, Bonnet F, Fromenty B (2013) Mitochondrial adaptations and dysfunctions in nonalcoholic fatty liver disease. Hepatology 58:1497-1507

34. Sunny NE, Kalavalapalli S, Bril F et al (2015) Cross-talk between branched-chain amino acids and hepatic mitochondria is compromised in nonalcoholic fatty liver disease. Am J Physiol Endocrinol Metab 309:E311-E319

35. Patterson RE, Kalavalapalli S, Williams CM et al (2016) Lipotoxicity in steatohepatitis occurs despite an increase in tricarboxylic acid cycle activity. Am J Physiol Endocrinol Metab. doi: 10.1152/ajpendo.00492.2015

36. Perry RJ, Zhang D, Zhang XM, Boyer JL, Shulman GI (2015) Controlled-release mitochondrial protonophore reverses diabetes and steatohepatitis in rats. Science 347:1253-1256

37. Satapati S, Kucejova B, Duarte JA et al (2015) Mitochondrial metabolism mediates oxidative stress and inflammation in fatty liver. $\mathrm{J}$ Clin Invest 125:4447-4462 
38. Koliaki C, Szendroedi J, Jelenik T et al (2015) Adaptation of hepatic mitochondrial function in humans with non-alcoholic fatty liver or steatohepatitis. Cell Metab 21:739-746

39. Sunny NE, Parks EJ, Browning JD, Burgess SC (2011) Excessive hepatic mitochondrial TCA cycle and gluconeogenesis in humans with nonalcoholic fatty liver disease. Cell Metab 14:804-810

40. Finan B, Yang B, Ottaway N et al (2015) A rationally designed monomeric peptide triagonist corrects obesity and diabetes in rodents. Nat Med 21:27-36

41. Soccio RE, Chen ER, Lazar MA (2014) Thiazolidinediones and the promise of insulin sensitization in type 2 diabetes. Cell Metab 20: 573-591

42. Belfort R, Harrison SA, Brown K et al (2006) A placebo-controlled trial of pioglitazone in subjects with nonalcoholic steatohepatitis. N Engl J Med 355:2297-2307

43. Gastaldelli A, Harrison S, Belfort R et al (2009) Importance of changes in adipose tissue insulin resistance to histological response during thiazolidinedione treatment of patients with nonalcoholic steatohepatitis. Hepatology 50:1087-1093

44. Gastaldelli A, Harrison S, Belfort-Aguiar R et al (2010) Pioglitazone in the treatment of NASH: the role of adiponectin. Aliment Pharmacol Ther 32:769-775

45. Bril F, Portillo Sanchez P, Maximos M et al (2015) Metabolic predictors of response to pioglitazone treatment in patients with prediabetes or type 2 diabetes mellitus and nonalcoholic steatohepatitis. Diabetes 64:A337 (Abstract)

46. DeFronzo RA, Tripathy D, Schwenke DC et al (2011) Pioglitazone for diabetes prevention in impaired glucose tolerance. N Engl J Med 364:1104-1115

47. Chang Y, Jung HS, Yun KE, Cho J, Cho YK, Ryu S (2013) Cohort study of non-alcoholic fatty liver disease, NAFLD fibrosis score, and the risk of incident diabetes in a Korean population. Am J Gastroenterol 108:1861-1868

48. Aithal GP, Thomas JA, Kaye PV et al (2008) Randomized, placebocontrolled trial of pioglitazone in nondiabetic subjects with nonalcoholic steatohepatitis. Gastroenterology 135:1176-1184

49. Sanyal AJ, Chalasani N, Kowdley KV et al (2010) Pioglitazone, vitamin E, or placebo for nonalcoholic steatohepatitis. N Engl J Med 362:1675-1685

50. Berria R, Gastaldelli A, Lucidi S et al (2006) Reduction in hematocrit level after pioglitazone treatment is correlated with decreased plasma free testosterone level, not hemodilution, in women with polycystic ovary syndrome. Clin Pharmacol Ther 80:105-114

51. Balas B, Belfort R, Harrison S et al (2007) Pioglitazone treatment increases whole body fat but not total body water in patients with non-alcoholic steatohepatitis. J Hepatol 47:565-570

52. VanWagner LB, Wilcox JE, Colangelo LA, Lloyd-Jones DM, Carr JJ, Lima JA (2015) Association of nonalcoholic fatty liver disease with subclinical myocardial remodeling and dysfunction: a population-based study. Hepatology 62:773-783

53. Yau H, Rivera K, Lomonaco R, Cusi K (2013) The future of thiazolidinedione therapy in the management of type 2 diabetes mellitus. Curr Diab Rep 13:329-341

54. Lewis JD, Habel LA, Quesenberry CP et al (2015) Pioglitazone use and risk of bladder cancer and other common cancers in persons with diabetes. JAMA 314:265-277

55. Portillo-Sanchez P, Cusi K (2016) Treatment of nonalcoholic fatty liver disease (NAFLD) in patients with type 2 diabetes mellitus. Clin Diab Endocrinol (in press)

56. Armstrong MJ, Houlihan DD, Rowe IA et al (2013) Safety and efficacy of liraglutide in patients with type 2 diabetes with elevated liver enzymes: individual patient data meta-analysis of the LEAD programme. Aliment Pharmacol Ther 37:234-242

57. Armstrong MJ, Gaunt P, Aithal GP et al (2016) Liraglutide safety and efficacy in patients with non-alcoholic steatohepatitis (LEAN): a multicentre, double-blind, randomised, placebo-controlled phase 2 study. Lancet 387:679-690

58. Armstrong MJ, Hull D, Guo K, Barton D, Hazlehurst JM, Gathercole LL (2016) Glucagon-like peptide 1 decreases lipotoxicity in non-alcoholic steatophepatitis. J Hepatol 64:399 408

59. Portillo P, Yavuz S, Bril F, Cusi K (2014) Role of insulin resistance and diabetes in the pathogenesis and treatment of nonalcoholic fatty liver disease. Curr Hepatol Rep 13:159-170

60. Gupta NA, Mells J, Dunham RM et al (2010) Glucagon-like peptide-1 receptor is present on human hepatocytes and has a direct role in decreasing hepatic steatosis in vitro by modulating elements of the insulin signaling pathway. Hepatology 51:1584-1592

61. Svegliati-Baroni G, Saccomanno S, Rychlicki C, Agostinelli L, De Minicis S, Candelaresi C (2011) Glucagon-like peptide-1 receptor activation stimulates hepatic lipid oxidation and restores hepatic signalling alteration induced by a high-fat diet in nonalcoholic steatohepatitis. Liver Int 31:1285-1297

62. Iwasaki T, Yoneda M, Inamori $M$ et al (2011) Sitagliptin as a novel treatment agent for non-alcoholic fatty liver disease patients with type 2 diabetes mellitus. Hepatogastroenterology 58:2103-2105

63. Fukuhara T, Hyogo H, Ochi H et al (2014) Efficacy and safety of sitagliptin for the treatment of nonalcoholic fatty liver disease with type 2 diabetes mellitus. Hepatogastroenterology 61:323-328

64. Macauley M, Hollingsworth KG, Smith FE et al (2015) Effect of vildagliptin on hepatic steatosis. J Clin Endocrinol Metab 100: $1578-1585$

65. Mudaliar S, Polidori D, Zambrowicz B, Henry RR (2015) Sodiumglucose cotransporter inhibitors: effects on renal and intestinal glucose transport: from bench to bedside. Diabetes Care 38:2344-2353

66. Hayashizaki-Someya Y, Kurosaki E, Takasu T et al (2015) Ipragliflozin, an SGLT2 inhibitor, exhibits a prophylactic effect on hepatic steatosis and fibrosis induced by choline-deficient Lamino acid-defined diet in rats. Eur J Pharmacol 754:19-24

67. Lavalle-Gonzalez FJ, Januszewicz A, Davidson J et al (2013) Efficacy and safety of canagliflozin compared with placebo and sitagliptin in patients with type 2 diabetes on background metformin monotherapy: a randomised trial. Diabetologia 56:2582-2592

68. Bailey CJ, Gross JL, Pieters A, Bastien A, List JF (2010) Effect of dapagliflozin in patients with type 2 diabetes who have inadequate glycaemic control with metformin: a randomised, double-blind, placebo-controlled trial. Lancet 375:2223-2233

69. Leiter LA, Forst T, Polidori D, Balis DA, Xie J, Sha S (2016) Effect of canagliflozin on liver function tests in patients with type 2 diabetes. Diabetes Metab 42:25-32

70. Nelson A, Torres DM, Morgan AE, Fincke C, Harrison SA (2009) A pilot study using simvastatin in the treatment of nonalcoholic steatohepatitis: a randomized placebo-controlled trial. J Clin Gastroenterol 43:990-994

71. Loomba R, Sirlin CB, Ang B et al (2015) Ezetimibe for the treatment of nonalcoholic steatohepatitis: assessment by novel magnetic resonance imaging and magnetic resonance elastography in a randomized trial (MOZART trial). Hepatology 61:1239-1250

72. Fabbrini E, Mohammed BS, Korenblat KM et al (2010) Effect of fenofibrate and niacin on intrahepatic triglyceride content, very low-density lipoprotein kinetics, and insulin action in obese subjects with nonalcoholic fatty liver disease. J Clin Endocrinol Metab 95:2727-2735

73. Dasarathy S, Dasarathy J, Khiyami A et al (2015) Double-blind randomized placebo-controlled clinical trial of omega 3 fatty acids for the treatment of diabetic patients with nonalcoholic steatohepatitis. J Clin Gastroenterol 49:137-144

74. Le TA, Chen J, Changchien C et al (2012) Effect of colesevelam on liver fat quantified by magnetic resonance in nonalcoholic steatohepatitis: a randomized controlled trial. Hepatology 56:922932 
75. Klein EA, Thompson IM, Tangen CM et al (2011) Vitamin E and the risk of prostate cancer: updated results of the Selenium and Vitamin E Cancer Prevention Trial (SELECT). JAMA 306:15491556

76. Van Wagner LB, Koppe SW, Brunt EM et al (2011) Pentoxifylline for the treatment of non-alcoholic steatohepatitis: a randomized controlled trial. Ann Hepatol 10:277-286

77. Zein CO, Yerian LM, Gogate P et al (2011) Pentoxifylline improves nonalcoholic steatohepatitis: a randomized placebo-controlled trial. Hepatology 54:1610-1619

78. Stefan N, Ramsauer M, Jordan P et al (2014) Inhibition of $11 \beta$ HSD1 with RO5093151 for non-alcoholic fatty liver disease: a multicentre, randomised, double-blind, placebo-controlled trial. Lancet Diabetes Endocrinol 2:406-416

79. Safadi R, Konikoff FM, Mahamid M, Zelber-Sagi S, Halpern M, Gilat T (2014) The fatty acid-bile acid conjugate aramchol reduces liver fat content in patients with nonalcoholic fatty liver disease. Clin Gastroenterol Hepatol 12:2085-2091

80. Cariou B, Staels B (2014) GFT505 for the treatment of nonalcoholic steatohepatitis and type 2 diabetes. Expert Opin Investig Drugs 23:1441-1448

81. Staels B, Rubenstrunk A, Noel B et al (2013) Hepatoprotective effects of the dual peroxisome proliferator-activated receptor alpha/delta agonist, GFT505, in rodent models of nonalcoholic fatty liver disease/nonalcoholic steatohepatitis. Hepatology 58:19411952

82. Odegaard JI, Ricardo-Gonzalez RR, Red Eagle A et al (2008) Alternative M2 activation of Kupffer cells by PPAR $\delta$ ameliorates obesity-induced insulin resistance. Cell Metab 7:496-507

83. Cariou B, Hanf R, Lambert-Porcheron S et al (2013) Dual peroxisome proliferator-activated receptor $\alpha / \delta$ agonist GFT505 improves hepatic and peripheral insulin sensitivity in abdominally obese subjects. Diabetes Care 36:2923-2930

84. Belfort R, Berria R, Cornell J, Cusi K (2010) Fenofibrate reduces systemic inflammation markers independent of its effects on lipid and glucose metabolism in patients with the metabolic syndrome. $\mathrm{J}$ Clin Endocrinol Metab 95:829-836
85. Ratziu V, Harrison S, Francque SM et al (2016) Elafibranor, an agonist of the peroxisome proliferator-activated receptor- $\alpha$ and $-\delta$, induces resolution of non-alcoholic steatohepatitis without fibrosis worsening. Gastroenterology. doi:10.1053/j.gastro.2016.01.038

86. Lefebvre E, Hashiguchi T, Jenkins $\mathrm{H}$ et al (2013) Anti-fibrotic and anti-inflammatory activity of the dual CCR2 and CCR5 antagonist cenicriviroc in a mouse model of NASH. Hepatology 58(S1): 221A-222A (Abstract)

87. Krenkel O, Püngel T, Mossanen JC et al (2015) Dual CCR2 and CCR5 antagonist cenicriviroc leads to potent and significant reduction in proinflammatory CCR $2+$ monocyte infiltration in experimental acute liver injury. Hepatology 62(S1):1065 (Abstract no. 1756)

88. Friedman S, Sanyal A, Goodman Z et al (2016) Efficacy and safety study of cenicriviroc for the treatment of non-alcoholic steatohepatitis in adult subjects with liver fibrosis: CENTAUR Phase $2 \mathrm{~b}$ study design. Contemp Clin Trials 47:356-365

89. Traber PG, Zomer E (2013) Therapy of experimental NASH and fibrosis with galectin inhibitors. Plos One 8:e83481

90. Fisher FM, Chui PC, Nasser IA et al (2014) Fibroblast growth factor 21 limits lipotoxicity by promoting hepatic fatty acid activation in mice on methionine and choline-deficient diets. Gastroenterology 147:1073-1083

91. Jiang C, Xie C, Li F et al (2015) Intestinal farnesoid X receptor signaling promotes nonalcoholic fatty liver disease. J Clin Invest 125:386-402

92. Neuschwander-Tetri BA, Loomba R, Sanyal AJ et al (2015) Farnesoid X nuclear receptor ligand obeticholic acid for non-cirrhotic, non-alcoholic steatohepatitis (FLINT): a multicentre, randomised, placebo-controlled trial. Lancet 385:956-965

93. Mudaliar S, Henry RR, Sanyal AJ et al (2013) Efficacy and safety of the farnesoid $\mathrm{X}$ receptor agonist obeticholic acid in patients with type 2 diabetes and nonalcoholic fatty liver disease. Gastroenterology 145:574-582

94. Neuschwander-Tetri BA (2010) Hepatic lipotoxicity and the pathogenesis of nonalcoholic steatohepatitis: the central role of nontriglyceride fatty acid metabolites. Hepatology 52:774-788

95. Dufour J-F (2016) Time to abandon NASH? Hepatology 63:9-10 